\title{
Primary gastrointestinal stromal tumor of the liver: A case report and review of the literature
}

\author{
XIAOBIN CHENG, DONG CHEN, WENBIN CHEN and QINSONG SHENG \\ Department of Colorectal Surgery, The First Affiliated Hospital, School of Medicine, \\ Zhejiang University, Hangzhou, Zhejiang 310003, P.R. China
}

Received March 10, 2015; Accepted March 18, 2016

DOI: $10.3892 / \mathrm{ol} .2016 .4981$

\begin{abstract}
Gastrointestinal stromal tumors (GISTs) are the most common mesenchymal tumors located in the alimentary tract. A small portion of GISTs are observed in extra-gastrointestinal regions, primarily in the omentum, mesentery and retroperioneum, and these types of GISTs are referred to as extra-gastrointestinal stromal tumors. The present study reports of a patient with unique primary liver GIST. The patient underwent en bloc resection and post-operative administration of imatinib, and subsequently experienced a good prognosis. The present case is followed by a brief review of reported cases of liver GISTs identified in the literature. The literature revealed that primary liver GISTs are usually large in size and possess a high mitotic index, which contributes to malignant characterization, thus classifying these tumors as high-risk. En bloc resection remains the mainstay of treatment for resectable primary liver GISTs. However, the prognosis of these patients is not favorable. Perioperative administration of imatinib may be useful to a certain extent, and interventional therapy, including radiofrequency ablation, should be considered.
\end{abstract}

\section{Introduction}

Gastrointestinal stromal tumors (GISTs) are rare tumors located in the alimentary tract, which originate from mesenchymal tissues, and have gene mutations, most often mast/stem cell growth factor receptor (CD117) and platelet-derived growth factor receptor- $\alpha$ (1). In Europe, the estimated annual incidence of clinically detected GISTs is 10 cases per million individuals, and the age-adjusted incidence is 7 cases per million individuals in Europe and the USA. However, at

Correspondence to: Dr Qinsong Sheng, Department of Colorectal Surgery, The First Affiliated Hospital, School of Medicine, Zhejiang University, 79 Qingchun Road, Hangzhou, Zhejiang 310003, P.R. China

E-mail: 20918146@zju.edu.cn

Key words: gastrointestinal stromal tumors, liver, primary, review, report present the global incidence of GISTs remains unknown (2). GISTs may arise anywhere along the gastrointestinal tract, but are identified primarily in the stomach (50-60\%) and small intestine (30-35\%), and less frequently in the colon and rectum $(5 \%)$ and oesophagus $(<1 \%)$ (3). Previously, a few cases of GISTs were diagnosed outside the gastrointestinal tract, usually in the omentum, mesentery or retroperioneum (4). This type of GIST is referred to as an extra-gastrointestinal stromal tumor (EGIST), which contributes to $<5 \%$ of all GISTs (3). In addition, to the tumor size and mitotic index, the malignant behavior of a GIST is associated with the primary location (3). EGISTs possess a higher malignant potential and risk of recurrence following surgery compared with GISTs in the alimentary tract (3). Other unusual primary anatomic locations of GISTs have also been reported, including the liver (5), pancreas (6), mediastinum (7) and gall bladder (8). The standard treatment for resectable GISTs is en bloc resection. The majority of patients may also benefit from perioperative administration of tyrosine kinase inhibitors (2). The estimated 5- and 15-year recurrence-free survival rates for GISTs treated with surgery alone are 70.5 and $59.9 \%$, respectively (2). As with GIST, complete surgical resection is the primary treatment for EGIST. However, compared with GIST, EGIST is considered to exhibit a worse prognosis (9). To the best of our knowledge, only 10 patients with primary liver GIST had been reported in the world (5,10-18). The present study reports a novel case of primary liver GIST and reviews the literature concerning all primary liver GISTs previously reported. Written informed consent was obtained from the patient.

\section{Case report}

A 63-year-old man was admitted to The First Affiliated Hospital of Zhejiang University (Hangzhou, China) on September 5, 2011, with a liver mass, which was detected by ultrasound. No clinical manifestations were present, including abdominal pain and distention, nausea and vomiting. The patient had suffered from hypertension for 10 years, which was well regulated with oral plendil $(5 \mathrm{mg})$ taken daily. A cholecystectomy was performed 12 years ago, due to gallbladder polyps, and an appendectomy was performed 25 years ago, due to acute appendicitis. A review of the medical history of the patient excluded systemic disease and any history of other malignancies. Laboratory values were within normal limits. 
Serum concentration of tumor markers revealed the following: Alpha-fetoprotein, $2.3 \mathrm{ng} / \mathrm{ml}$ (normal range, $<20.0 \mathrm{ng} / \mathrm{ml}$ ); carcinoembryonic antigen, $2.2 \mathrm{ng} / \mathrm{ml}$ (normal range, $<5.0 \mathrm{ng} / \mathrm{ml}$ ); CA125, 8.0 U/ml (normal range, $<35.0 \mathrm{U} / \mathrm{ml}$ ); and CA199, $23.0 \mathrm{U} / \mathrm{ml}$ (normal range, $<37.0 \mathrm{U} / \mathrm{ml}$ ).

Abdominal contrast-enhanced computed tomography (CT) scan (Aquilion 16; Toshiba, Tokyo, Japan) revealed the presence of a cystic-solid mixed mass with a suspected internal hemorrhage in the right liver lobe (Fig. 1). The mass was measured at $11 \times 13 \times 15 \mathrm{~cm}$ at the widest point. The peripheral margin was irregular and thickened with heterogeneous enhancement on the CT scan. No tumors were detected in other organs, with the exception of multiple cysts in the liver and left kidney. A right hepatectomy was performed to remove the tumor completely on September 20, 2011. On gross examination, the tumor contained numerous blood clots. Microscopically (DM2500; Leica Microsystems, Wetzlar, Germany), the tumor cells were atypical and spindle-shaped. There was a high cellular density and infiltrative growth was observed. The mitotic count was $>5 / 50$ high power fields (HPF). Resected tissues were formalin-fixed, paraffinembedded and cut into 4-mm sections. The sections were incubated with primary monoclonal mouse anti-human CD34 (cat. no. M0117; 1:200; ChangDao, Shanghai, China) and monoclonal mouse anti-human CD117 (cat. no. ZM0437; 1:200; Zhongshan Golden Bridge Biotechnology Co., Ltd., Beijing, China) at $4^{\circ} \mathrm{C}$ overnight. Following three washes with phosphate-buffered saline (PBS), the sections were incubated with ready-to-use horseradish peroxidase-conjugated goat anti-mouse IgG secondary antibody (cat. no. A0216; 1:50; Beyotime Institute of Biotechnology, Shanghai, China) for $20 \mathrm{~min}$ at $37^{\circ} \mathrm{C}$. After three washes with PBS (Beyotime Institute of Biotechnology), the sections were then incubated with diaminobenzidine (Beyotime Institute of Biotechnology) substrate for $5 \mathrm{~min}$ at room temperature and staining was visualized using a microscope (DM2500; Leica Microsystems). Immunohistochemistry revealed positive staining for cluster of differentiation (CD) 34 and CD117 (Fig. 2). The immunohistochemistry results lead to a diagnosis of GIST. A liver metastatic lesion was considered and a positron emission tomography-CT was administered to detect the latent primary lesion. However, no tumor signs were observed and a consultation with the Department of Pathology and Radiology resulted in a diagnosis of primary liver GIST.

The post-operative recovery of the patient was uneventful. Adjuvant therapy of imatinib (400 mg/day, oral administration) was initiated one month subsequent to surgery, which lasted for two years. For follow-up, the patients underwent abdominal CT three times a year. After 5 years of follow-up, there was no evidence of tumor recurrence, and the patient is currently alive and well.

\section{Discussion}

A literature review performed by the present study revealed a total of 11 cases diagnosed with primary liver GIST worldwide, including the current case (Table I) $(5,10-18)$. In these studies, primary liver GISTs exhibited a male predominance to females (72.7 vs. $27.3 \%$ ), and the median age of occurrence was 63 years (range, 17-79 years). Similarly to GISTs located

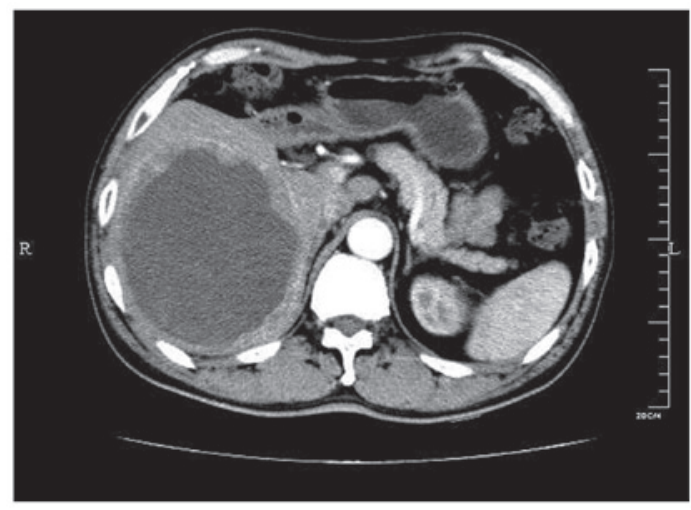

Figure 1. Abdominal contrast-enhanced computed tomography demonstrated the presence of a large cystic-solid mixed mass with suspected internal hemorrhage in the right lobe of the liver.
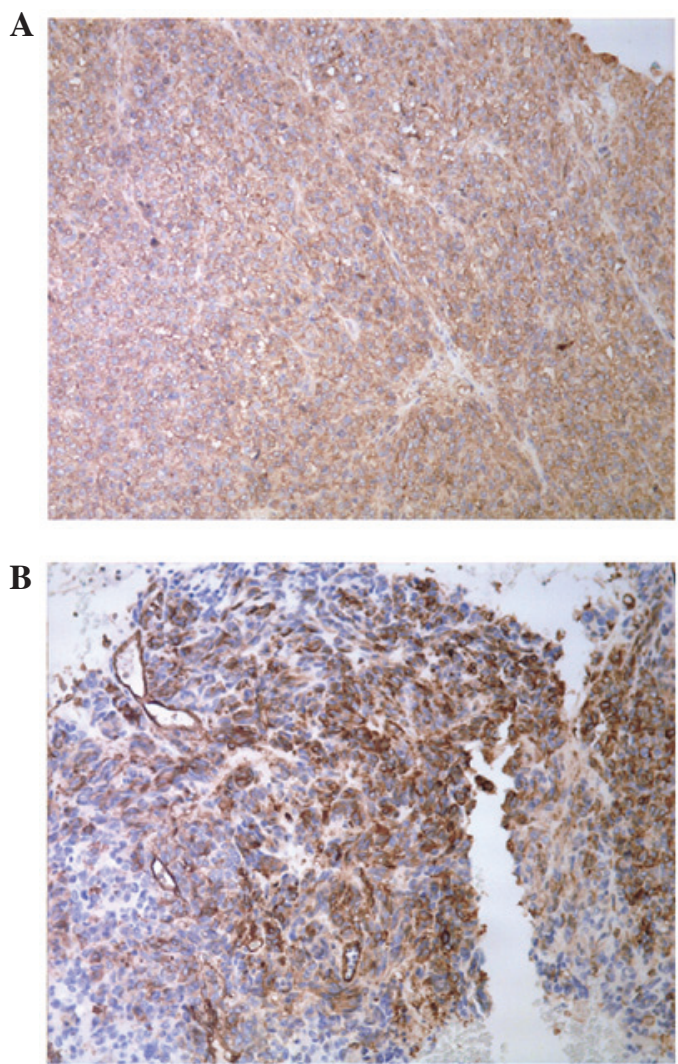

Figure 2. Immunohistochemical staining of the resected gastrointestinal stromal tumor of the liver for (A) mast/stem cell growth factor receptor and (B) cluster of differentiation 34. Magnification, x200.

in other organs, primary liver GISTs have no specific clinical manifestation, such as abdominal distention and pain, and shortness of breath (possibly occurring due to upward displacement of the diaphragm) may be the initial clinical manifestation owing to the large size of the primary liver lesions. Liver GISTs may not be identified if they are smaller in size, and require identification by magnetic resonance, computed tomography or ultrasound imaging. Microscopically, 10 out of the 11 cases reported data concerning cell morphology, which revealed that 7 tumors were of a spindle type, 1 was of a epithelioid type and 2 had spindle and epithelioid cells. The majority of the tumors in the 11 cases were of a large volume $(>10 \mathrm{~cm})$ 


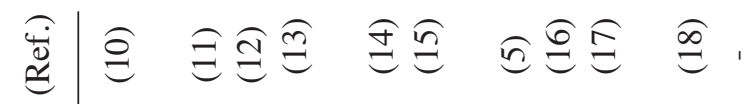

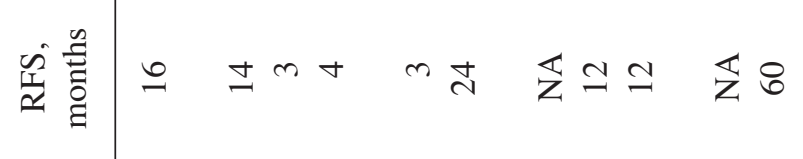

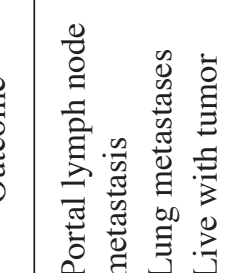

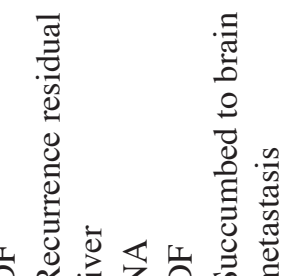

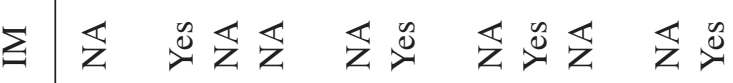

苞

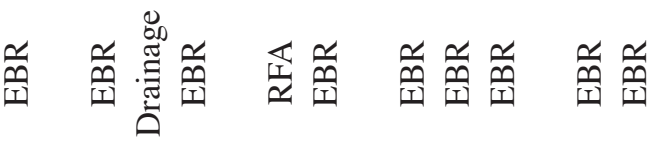

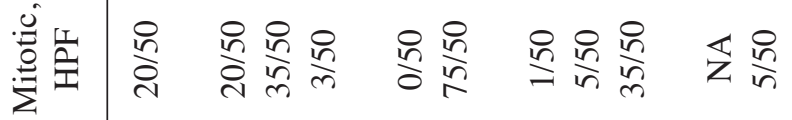

离

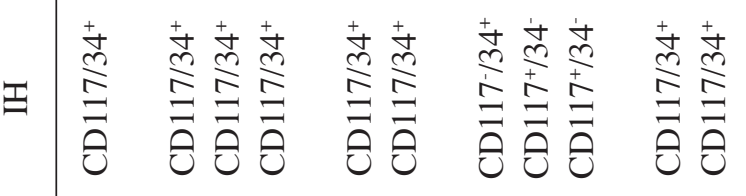

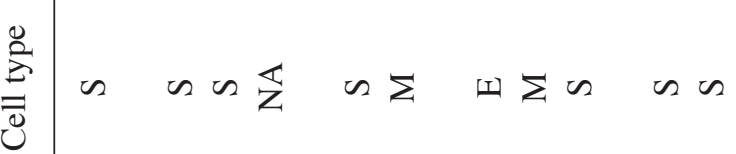

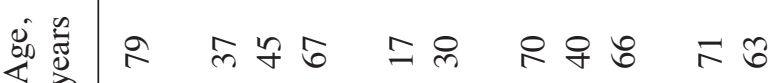

空导

-

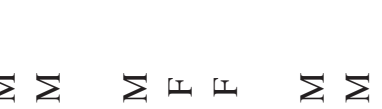

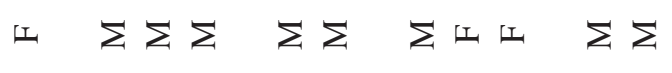

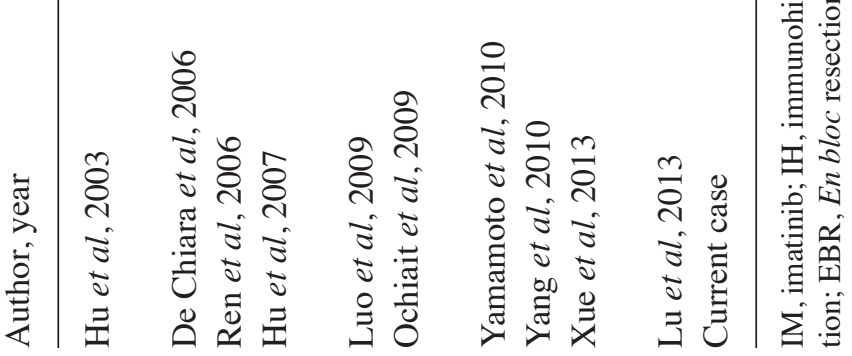


and 1 patient with a small tumor, and the median diameter of $18.0 \mathrm{~cm}$ (range, 5.1-44.0 cm). In addition, cells in the mitotic phase was commonly detected. Excepting 1 case without any mitosis and one case with no data, the median mitotic index was 20/50 HPF (range, 1-75/50 HPF). Immunohistochemical results revealed positive staining for CD117 (10/11) and CD34 (9/11) in the majority of the primary liver GIST cases, except 1 case and 2 cases that presented with negative staining for CD117 and CD34, respectively.

The majority of the patients were defined as possessing high-risk GIST, according to the National Institute of Health criteria (19), except for 1 case that was classed as low-risk. Curative surgery remains the mainstay of treatment for resectable high-risk lesions, and 9 out of 11 patients underwent resection of the tumor. In total, 4 patients received adjuvant therapy with imatinib post-operatively. Of the 11 patients, prognostic data on 7 patients were reported: 3 patients had no recurrent signs during follow-up; 1 patient underwent recurrence twice at 2 and 5 years following the first surgery, followed by a second and third surgery with adjuvant imatinib treatment, and the patient was alive with no recurrence at the end of follow-up; 1 patient presented with lung metastasis 14 months post-surgery and received imatinib, which lead to the metastasis becoming reduced and the patient had disease-free survival for the following 39 months; 1 patient presented with portal lymph node metastases 16 months post-surgery, and received a second curative surgery with no recurrence in subsequent follow-ups; 1 patient succumbed to brain metastasis 1 year post-operatively. For the above 7 patients, the median recurrence-free survival was 16 months (95\% confidence interval, 11.8-20.2 months; range, 4.0-60.0 months). Radiofrequency ablation (RFA) was administered to the patient with the small low-risk lesion. This patient recovered quickly and had no recurrence during 3 months of follow-up. There was 1 patient with an unresectable high-risk lesion, which appeared to be a thick-walled cyst. The patient received percutaneous transhepatic drainage and nutritional support, and was alive with the tumor during 3 months of follow-up.

Primary liver GISTs are extremely uncommon compared to their alimentary counterparts, and EGISTs are gradually being diagnosed more regularly, with lesions in the omentum, mesentery and retroperioneum already described (4). The current study reported a case of a male patient with a large primary liver GIST confirmed by immunohistochemistry and CT imaging. The high mitotic index and large tumor size contributed to a malignant characterization, thus classifying the tumor as high-risk. The patient received en bloc resection and post-operative adjuvant treatment of imatinib for 2 years. The patient had a good prognosis without recurrence in 5-years of follow-up. Primary liver GISTs are extremely rare; therefore, the possibility of metastasis in the current case was excluded. The present authors hypothesize that the current case is a true primary liver GIST for the following reasons: The lesion was completely limited to the liver; a CT scan and intraoperative exploration excluded the possibility of another primary lesion; and the patient had no history of malignancies, thus excluding the possibility of recurrence.

There are certain hypotheses concerning the origin of EGISTs. Since Min and Leabu (20) confirmed the presence of interstitial cells of Cajal (ICC) in the extra-digestive tract, including in the urinary bladder, gall bladder, uterus, omentum, prostate and myocardium, it is reasonable to presume that EGISTs originate from common precursor cells of ICC. Other authors suggest that EGISTs may originate from pluripotent mesenchymal stem cells located outside alimentary tract (9). Consequently, these observations may demonstrate the origin and existence of primary liver GISTs.

During an extensive literature retrieval, the present study identified 11 cases of primary liver GIST (5,10-18), including the current case. Similarly to alimentary GISTs, primary liver GISTs possess a male predilection and have no distinguishing symptoms, such as the presence of a swollen abdomen. The liver lesion may become large in size, since there is no evident discomfort or clinical manifestation in the early stages. Therefore, primary liver GISTs are often identified when they are large, while small lesions may be observed during periodical examination. In addition, primary liver GISTs possess clear proliferative activity, leading to high-risk classification in numerous primary liver GISTs. Immunohistochemically, the majority of primary liver lesions reveal positive staining for CD117. Overall, regarding the clinicopathological information available, primary liver GISTs are similar to conventional GISTs that originate in the alimentary tract.

Curative surgery remains the primary treatment option for resectable primary liver lesions. However, the prognosis of patients is not favorable, and 4 out of 7 patients identified in the literature search with post-operative data had recurrence. This may be due to the strong proliferative activity of primary lesions and potential satellite lesions, as described by $\mathrm{Hu}$ et al (10). Furthermore, EGISTs have been reported to be frequently accompanied by adverse prognostic factors, including a high proliferative index, large size, lymph node involvement and distant metastasis (9). Consequently, patients with EGISTs are considered to have a poorer prognosis compared to alimentary GISTs (9). Imatinib treatment may be considered preoperatively in order to inhibit tumor activity, and works by minimizing the tumor load and eliminating potential satellite lesions (21). Post-operatively, imatinib may be administered for adjuvant treatment for patients with a high recurrence risk, which is similar to the guidelines for their alimentary counterparts (22). Although imatinib exhibits a satisfactory curative effect in certain cases $(11,16)$, the clinical value of imatinib on primary liver GISTs requires verification. Previously, a mutational analysis of EGISTs reported by Yamamoto et al (4) revealed an infrequent mutation of CD117 on exon 11, which suggests a good response to imatinib (9). Only 1 case among the primary liver GISTs reported has been identified with a platelet-derived growth factor receptor, alpha polypeptide exon 12 mutation and 1 case with a CD117 exon 11 mutation $(5,15)$. Deficient data on the analysis of gene mutations in GISTs impedes the understanding of the behavior and intervention therapeutics for this disease. RFA, hepatic arterial embolization or chemoembolization are usually administered for the management of liver metastases of GISTs $(23,24)$. Following the identification of primary liver GISTs, these techniques may also be considered for unresectable lesions. Notably, RFA may be a useful and minimally invasive interventional therapy for curative treatment of small lesions (14). 
In conclusion, the present study described a case of primary liver GIST, and discussed the associated immunohistochemical and molecular features, in addition to treatment options. En bloc resection remains the optimal treatment for resectable masses. The value of imatinib and other intervention therapeutics requires further verification via future investigation.

\section{Acknowledgements}

This study was supported by the Natural Science Foundation of Zhejiang Province (grant no. LY13H030004).

\section{References}

1. Kitamura Y: Gastrointestinal stromal tumors: Past, present and future. J Gastroenterol 43: 499-508, 2008.

2. Joensuu H, Hohenberger P and Corless CL: Gastrointestinal stromal tumour. Lancet 382: 973-983, 2013.

3. Joensuu H, Vehtari A, Riihimäki J, Nishida T, Steigen SE, Brabec P, Plank L, Nilsson B, Cirilli C, Braconi C, et al: Risk of recurrence of gastrointestinal stromal tumour after surgery: An analysis of pooled population-based cohorts. Lancet Oncol 13: 265-274, 2012.

4. Yamamoto H, Oda Y, Kawaguchi K, Nakamura N, Takahira T, Tamiya S, Saito T, Oshiro Y, Ohta M, Yao T and Tsuneyoshi M: c-kit and PDGFRA mutations in extragastrointestinal stromal tumor (gastrointestinal stromal tumor of the soft tissue). Am J Surg Pathol 28: 479-488, 2004.

5. Yamamoto H, Miyamoto Y, Nishihara Y, Kojima A, Imamura M, Kishikawa K, Takase Y, Ario K, Oda Y and Tsuneyoshi M: Primary gastrointestinal stromal tumor of the liver with PDGFRA gene mutation. Hum Pathol 41: 605-609, 2010.

6. Vij M, Agrawal V and Pandey R: Malignant extra-gastrointestinal stromal tumor of the pancreas. A case report and review of literature. JOP 12: 200-204, 2011.

7. Lee JR, Anstadt MP, Khwaja S and Green LK: Gastrointestinal stromal tumor of the posterior mediastinum. Eur J Cardiothorac Surg 22: 1014-1016, 2002.

8. Park JK, Choi SH, Lee S, Min KO, Yun SS and Jeon HM: Malignant gastrointestinal stromal tumor of the gallbladder. J Korean Med Sci 19: 763-767, 2004.

9. Yi JH, Sim J, Park BB, Lee YY, Jung WS, Jang HJ, Ha TK and Paik SS: The primary extra-gastrointestinal stromal tumor of pleura: A case report and a literature review. Jpn J Clin Oncol 43: 1269-1272, 2013.

10. Hu X, Forster J and Damjanov I: Primary malignant gastrointestinal stromal tumor of the liver. Arch Pathol Lab Med 127: 1606-1608, 2003.
11. De Chiara A, De Rosa V, Lastoria S, Franco R, Botti G, Iaffaioli VR and Apice G: Primary gastrointestinal stromal tumor of the liver with lung metastases successfully treated with STI-571 (imatinib mesylate). Front Biosci 11: 498-501, 2006.

12. Ren SY, Huang, ZQ and Dong BZ: Primary gastrointestinal stromal tumor of the liver: One case report. Zhonghua Yi Xue Za Zhi 76: 3311, 2006 (In Chinese).

13. Hu ZQ, Wei YS, Zhu HH, Yang Z, Deng ZZ, You M and Xiang Z: Huge malignant liver mesenchymal tumor: One case report. Zhongguo Shi Yong Wai Ke Za Zhi 27: 417, 2007 (In Chinese).

14. Luo XL, Liu D, Yang JJ, Zheng MW, Zhang J and Zhou XD: Primary gastrointestinal stromal tumor of the liver: A case report. World J Gastroenterol 15: 3704-3707, 2009.

15. Ochiai T, Sonoyama T, Kikuchi S, et al: Primary large gastrointestinal stromal tumor of the liver: Report of a case. Surg Today 39: 633-636, 2009.

16. Yang Y, Xing CP, Liu B, Su QJ and Dong L: Diagnosis and differential diagnosis of primary liver gastrointestinal stromal tumors. Modern Oncology 18: 2432-420, 2010.

17. Xue YJ and Hu XJ: Primary gastrointestinal stromal tumor of the liver: One case report. Zhong Liu Xue Za Zhi 19: 159-160, 2013 (In Chinese).

18. Lu Y and Guo SL: One case: Primary malignant stromal tumor of the liver. Shi Yong Fang She Xue Za Zhi 29: 1368-1369, 2013 (In Chinese).

19. Joensuu H: Risk stratification of patients diagnosed with gastrointestinal stromal tumor. Hum Pathol 39: 1411-1419, 2008 .

20. Min KW and Leabu M: Interstitial cells of Cajal (ICC) and gastrointestinal stromal tumor (GIST): Facts, speculations and myths. J Cell Mol Med 10: 995-1013, 2006.

21. Wilkinson MJ, Fitzgerald JE, Strauss DC, Hayes AJ, Thomas JM, Messiou C, Fisher C, Benson C, Tekkis PP and Judson I: Surgical treatment of gastrointestinal stromal tumour of the rectum in the era of imatinib. Br J Surg 102: 965-971, 2015.

22. von Mehren M, Randall RL, Benjamin RS, Boles S, Bui MM, Casper ES, Conrad EU III, DeLaney TF, Ganjoo KN, George S, et al: Gastrointestinal stromal tumors, version 2.2014. J Natl Compr Canc Netw 12: 853-862, 2014.

23. Kobayashi K, Szklaruk J, Trent JC, Ensor J, Ahrar K, Wallace MJ, Madoff DC, Murthy R, Hicks ME and Gupta S: Hepatic arterial embolization and chemoembolization for imatinib-resistant gastrointestinal stromal tumors. Am J Clin Oncol 32: 574-581, 2009.

24. Jones RL, McCall J, Adam A, O'Donnell D, Ashley S, Al-Muderis O, Thway K, Fisher C and Judson IR: Radiofrequency ablation is a feasible therapeutic option in the multi modality management of sarcoma. Eur J Surg Oncol 36: 477-482, 2010. 